markers, leptin measurement might assist in monitoring disease activity levels in patients with RA.

Original article Lee S-W et al. (2007) Measurement of the serum leptin level could assist disease activity monitoring in rheumatoid arthritis. Rheumatol Int 27: 537-540

\section{Patellar malalignment is associated with radiographic features of knee OA}

Patellar malalignment places excess stress on the patellofemoral joint, which can potentially lead to knee damage-a risk factor for the development of knee OA. Patellar alignment is usually assessed radiographically, but can also be assessed by transverse and sagittal $\mathrm{MRI}$ scans. Currently, knee OA is assessed by radiographic evaluation of joint-space narrowing (JSN) and osteophytes, and patellar alignment is typically not considered. Kalichman et al., therefore, investigated whether patellar alignment evaluated by MRI correlates with common radiographic indices of patellofemoral OA.

This cross-sectional, observational study included 213 participants (age range 4793 years, 126 males) with symptomatic knee OA randomly selected from the Boston Osteoarthritis of the Knee Study (a natural history study of symptomatic knee OA); 75\% of the group had tibiofemoral OA. MRI scans from the participants were analyzed to evaluate patellar alignment. After adjustment for age, sex and BMI, increases in patellar length ratio (calculated from sagittal plane measurements), lateral patellar tilt angle and bisect offset (both calculated from transverse plane measurements) were associated with increased JSN and osteophytosis in the lateral compartment. Increased sulcus angle was associated with medial JSN and lateral and medial patellar osteophytosis.

The MRI scans were obtained in supine patients. The authors acknowledge that some measures of patellar alignment might be different in a weight-bearing position or with the knees flexed and, therefore, further studies that investigate these associations could use functional knee positions.

Original article Kalichman L et al. (2007) The association between patella alignment on magnetic resonance imaging and radiographic manifestations of knee osteoarthritis. Arthritis Res Ther 9: R26

\section{Potential use for IL-1 blockade in the treatment of gout}

Several agents are available and generally effective for the treatment of gout; however, many are poorly tolerated, or contraindicated in patients with renal, cardiovascular or gastrointestinal disease. Studies have shown that monosodium urate crystals induce interleukin-1 (IL-1) production, by activation of the NALP3 (cryopyrin) inflammasome, which led investigators from Switzerland to determine whether IL-1 blockade could inhibit inflammation induced by monosodium urate crystals.

Tschopp and colleagues used a mouse model of monosodium-urate-induced peritoneal inflammation to show that neutrophil recruitment was inhibited in the lavage fluids of mice treated with either an IL-1 monoclonal antibody, or the anti-IL-1 agent anakinra. This effect was not observed in mice treated with a tumor necrosis factor monoclonal antibody.

These investigators also conducted an open-label trial of anakinra in 10 patients with acute gouty arthritis that was refractory to (or who could not tolerate) conventional gout treatment. Patients received anakinra $100 \mathrm{mg}$ daily subcutaneously for 3 days. Colchicine or NSAID treatment was discontinued, but lowdose corticosteroids were continued at the same dose if previously administered. Patients were followed up for $>1$ month, during which no adverse effects were observed. All patients responded rapidly to anakinra, and their subjective symptoms of gout were relieved within $48 \mathrm{~h}$ after the first injection. Complete resolution of signs of arthritis was observed in nine patients.

The authors stress that the study was an open-label trial, and that randomized controlled trials are required to demonstrate that IL-1 inhibition is effective for treating acute gout.

Original article So A et al. (2007) A pilot study of IL-1 inhibition by anakinra in acute gout. Arthritis Res Ther 9: R28

\section{Mycophenolate mofetil is a possible treatment for scleroderma}

Substantial evidence exists that patients with scleroderma have immune dysfunction and autoimmunity, and several studies support the 